This is the peer reviewed version of the following article: Wollenberg Valero, K.C., Garcia-Porta, J., Irisarri, I., Feugere, L., Bates, A., Kirchhof, S., Jovanović Glavaš, O., Pafilis, P., Samuel, S.F., Müller, J., Vences, M., Turner, A.P., Beltran-Alvarez, P. and Storey, K.B. (2021), Functional genomics of abiotic environmental adaptation in lacertid lizards and other vertebrates. Journal of Animal Ecology. Accepted Author Manuscript., which has been published in final form at https://doi.org/10.1111/13652656.13617. This article may be used for non-commercial purposes in accordance with Wiley Terms and Conditions for Use of Self-Archived Versions. This article may not be enhanced, enriched or otherwise transformed into a derivative work, without express permission from Wiley or by statutory rights under applicable legislation. Copyright notices must not be removed, obscured or modified. The article must be linked to Wiley's version of record on Wiley Online Library and any embedding, framing or otherwise making available the article or pages thereof by third parties from platforms, services and websites other than Wiley Online Library must be prohibited. 


\section{Functional genomics of abiotic environmental adaptation in lacertid lizards and other vertebrates}

Katharina C. Wollenberg Valero ${ }^{1 *}$, Joan Garcia-Porta ${ }^{2}$, Iker Irisarri ${ }^{3,4}$, Lauric Feugere ${ }^{1}$, Adam Bates ${ }^{1}$, Sebastian Kirchhof ${ }^{5,6}$, Olga Jovanović Glavaš ${ }^{7}$, Panayiotis Pafilis ${ }^{8}$, Sabrina F. Samuel ${ }^{9}$, Johannes Müller ${ }^{5}$, Miguel Vences ${ }^{10}$, Alexander P. Turner ${ }^{1}$, Pedro BeltranAlvarez $^{9}$, Kenneth B. Storey ${ }^{11}$

${ }^{1}$ Department of Biological and Marine Sciences, University of Hull, Cottingham Road HU6 7RX, Kingston-Upon-Hull, United Kingdom

${ }^{2}$ Department of Biology, Washington University in Saint Louis, St. Louis, MO 63130, USA.

${ }^{3}$ Department of Applied Bioinformatics, Institute for Microbiology and Genetics, University of Göttingen, Goldschmidstr. 1, 37077 Göttingen, Germany

${ }^{4}$ Campus Institut Data Science (CIDAS), Göttingen, Germany

${ }^{5}$ Museum für Naturkunde, Leibniz Institute for Evolution and Biodiversity Science, Invalidenstr. 43, 10115 Berlin, Germany

${ }^{6}$ New York University Abu Dhabi, Saadiyat Island Campus, Abu Dhabi, United Arab Emirates

${ }^{7}$ Department of Biology, University of Osijek, Cara Hadrijana 8A, Osijek, Croatia

${ }^{8}$ Section of Zoology and Marine Biology, Department of Biology, National and Kapodistrian University of Athens, Panepistimioupolis, Ilissia, Athens 157-84, Greece

${ }^{9}$ Department of Biomedical Sciences, University of Hull, Cottingham Road HU6 7RX, KingstonUpon-Hull, United Kingdom

${ }^{10}$ Zoological Institute, Braunschweig University of Technology, Mendelssohnstr. 4, 38106 Braunschweig, Germany

${ }^{11}$ Department of Biology, Carleton University, 1125 Colonel By Drive, K1S 5B6 Ottawa, Canada

*Author for Correspondence: Katharina Wollenberg Valero, Department of Biological and Marine Sciences, University of Hull, UK, +44 1482 465187, k.wollenberg-valero@hull.ac.uk

\section{Data Availability}

Additional figures, tables and code is provided in Supplementary Materials. RNA-Seq data are available from the Sequence Read Archive SRA Bioproject PRJNA543749. Additional transcriptomes and assemblies can be found in PRJNA310422 and two more are available at doi:10.6084/m9.figshare. 15010923 and doi: $10.6084 / \mathrm{m} 9$.figshare. 15010935 . The data table for environmental adaptation genes and functions is available at doi: $10.6084 / \mathrm{m} 9$.figshare. 15010887 and is available as an interactive resource at: www.cassandra-genes.org. A network cytoscape session with the vertebrate network as well as the ClueGo results is available at doi:10.6084/m9.figshare. 15010872 . Code is provided in the Supplementary Materials as Supplementary Appendix 1.

Manuscript type: Research Article 


\section{Abstract}

1. Understanding the genomic basis of adaptation to different abiotic environments is important in the context of climate change and resulting short-term environmental fluctuations.

2. Using functional and comparative genomics approaches, we here investigated whether signatures of genomic adaptation to a set of environmental parameters are concentrated in specific subsets of genes and functions in lacertid lizards and other vertebrates.

3. We first identify 200 genes with signatures of positive diversifying selection from transcriptomes of 24 species of lacertid lizards and demonstrate their involvement in physiological and morphological adaptations to climate. To understand how functionally similar these genes are to previously predicted candidate functions for climate adaptation and to compare them with other vertebrate species, we then performed a meta-analysis of 1100 genes under selection obtained from -omics studies in vertebrate species adapted to different abiotic environments.

4. We found that the vertebrate gene set formed a tightly connected interactome, which was $23 \%$ enriched in previously predicted functions of adaptation to climate, and to a large part (18\%) involved in organismal stress response. We found a much higher degree of identical genes being repeatedly selected among different animal groups (43.6\%), a higher degree of functional similarity, and posttranslational modifications than expected by chance, and no clear functional division between genes used for ectotherm and endotherm physiological strategies. 171 out of 200 genes of Lacertidae were part of this network.

5. These results highlight an important role of a comparatively small set of genes and their functions in environmental adaptation and narrows the set of candidate pathways and markers to be used in future research on adaptation and stress response related to climate change.

Keywords: environmental adaptation, functional genomics, comparative genomics, constraint, repeated positive diversifying selection 


\section{Introduction}

The sheer number of genes and alleles within a genome (Futuyma, 2010), the fact that many traits are polygenic (Turelli, 1985) and that genomes can evolve via alternative pathways (Salverda et al., 2011) theoretically provide infinite possibilities for adaptations to evolve. Because of this, genomes are expected to reflect a high degree of evolutionary unpredictability (Blount et al., 2018; Therkildsen et al., 2019). However, many realized adaptations seem less infinite than expected, which means that constraints at the genome level could lead to more predictable outcomes (Blount et al., 2018; Futuyma, 2010). One such element of constraint may be explained through the interaction of genes that perform a common function upon which selection acts, called gene functional constraint (Han et al., 2004; Pavlicev \& Wagner, 2012; Wollenberg Valero, 2020). This question of which subsets of protein-coding genes within a genome are realized to perform specific functions has practical relevance for understanding the genomic basis of response to a changing climate, which targets "physiology" as a multi-gene multi-function concept. In this paper, we address which functional subsets of the genome are used in different vertebrate species in adaptation to aspects of the abiotic environment, whether events of such adaptation are concentrated in a specific subset of genes, and to which extent these genes have known involvement in different aspects of the stress response, both at organismal and cellular levels. For this study, we include under the term "abiotic environment" different environmental conditions comprising thermal differences, gradients of latitudinal climate, altitude or season, sulfidic water, osmotic differences, desiccation, hypoxia, or wet-dry environmental gradients. The answers to these questions will help narrow down candidate genes and pathways that can serve as targets for future studies of climate change adaptation and stress response.

Under complete relaxation of gene functional constraint during macroevolution, any genes could perform climate adaptation-related functions across different organisms -and would have an equal chance of being under climate-mediated selection- but this is not a likely scenario (cf. Wollenberg Valero 2020). In contrast, adaptation to different or novel abiotic environments involves changes in traits, which, in similar environments, often converge (Losos, 2011). We expect to find adaptations to similar selective pressures to be concentrated in a subset of genes performing a limited set of functions 
across different organisms, due to ontogenetic constraints and common descent (Babonis \& Martindale, 2017; Han et al., 2004). Selection operating on a constrained set of genes (due to links with specific functions), is in this scenario more likely to act on the same genes repeatedly in independent selection events. For example, adaptation to climate in vertebrate ectotherms may be directed through a specific set of gene functions, with some evidence that similar genes are involved (Porcelli et al., 2015; Rodríguez et al., 2017; Wollenberg Valero, 2020; Wollenberg Valero et al., 2014). Under strong constraint, this can lead to repeated selection on the same genes in adaptation to similar environments in different species. One of the best-known examples of repeated selection through modification of identical genes in different species is altitude adaptation in the Himalayas. High-altitude yak, Tibetan humans and their dogs all show adaptations in the ADAM17 gene related to the physiological function of hypoxia tolerance (Qiu et al., 2012; Simonson, 2015) compared to their low-altitude relatives. Another example for repeated selection in distantly related lineages are the antifreeze glycoprotein genes in Antarctic notothenioid fish and Arctic cod. Notothenioids re-purposed the glycoprotein sequence from a different ancestral function (Chen et al., 1997), whereas the same functional gene sequence in Arctic cod evolved de novo from a non-coding genomic region (Zhuang et al., 2019).

While endothermic vertebrates (including humans) have been well studied from an -omics perspective (Hancock et al., 2008), the genomic basis of environmental adaptation in vertebrate ectotherms is generally understudied (Diele-Viegas \& Rocha, 2018). Only a few studies incorporated genome-wide scans for environmental adaptation-relevant genes to date (Campbell-Staton et al., 2017; Rodríguez et al., 2017; Sun et al., 2018; Wollenberg Valero et al., 2019; Yang et al., 2014). Where development in the majority of taxa occurs outside the parental body, climate can be a strong selective force for both cold (Feiner et al., 2018; Rodríguez et al., 2017) and heat (Sanger et al., 2018) stress adaptation, during early development. In vertebrate ectotherms, these functions contain genes involved in the conserved stress response (Denver, 2009), e.g., heat shock proteins (HSPs, Logan \& Buckley, 2015), but it is not yet known how general this involvement of genes adapting to environmental changes is in the organismal response to abiotic stressors. Other candidates for environmental adaptation are genes active in cancer due to their involvement in pathways that usually regulate homeostasis in response to abiotic environmental stressors (Hayes et al., 2020; Plotnikov et al., 2011; Tan et al., 2019). Furthermore, 
oxidative cellular stress often ends in apoptosis (Buttke \& Sandstrom, 1994) and consequently, genes related to the cellular pathways of apoptosis, stress and inflammation (dubbed "Zombie genes"; (Pozhitkov et al., 2017) could also be candidates for environmental adaptation. Functionally similar genes, such as those involved in homeostasis, can further be recognized through similar epiproteomic signals, such as protein post-translational modifications (PTMs; Jensen et al., 2002; J. Yu \& Auwerx, 2010).

In this study we first focus on the genomic basis of latitudinal climate adaptation in squamate reptiles of Old-World lizards (family Lacertidae). We use an RNA sequencing (RNAseq) data set we previously generated for phylogenomic analysis of this group (Garcia-Porta et al., 2019) to identify genes with signatures of positive diversifying selection across 24 species representing the lacertid phylogenetic diversity. This group of lizards shows physiological adaptations to a (partly latitudinal) climate gradient in preferred body temperature ( $\mathrm{T}_{\text {pref }}$ ) and evaporative water loss (IWL), as well as aligned variation in genome-wide substitution rates, which serves as evidence for latitudinal climate adaptation (GarciaPorta et al., 2019). In this study, we identify genes with signatures of selection in Lacertidae and identify how these genes are related to parameters of climate as well as climate-related phenotypic variables. Secondly, to identify the extent of constraint in function and repeated events of selection on the same genes in adaptation to the abiotic environment across vertebrates, we compare the functions of genes putatively involved in environmental adaptation (in the following called "putative environmental adaptation genes", pEAGs) across vertebrates, mined from 22 -omics studies of adaptation in vertebrates. Under the premise of gene functional constraint, we expect that pEAGs identified in different vertebrate lineages are tightly functionally connected and fall within predefined functions for climate adaptation (Porcelli et al., 2015; Rodríguez et al., 2017; Wollenberg Valero et al., 2014). We infer whether these genes are also up- or downregulated in the organismal response to abiotic stressors (by evaluating 75 mostly -omics studies of the response to stress in animals including non-vertebrates). We further explore the functions of pEAG through inferring whether they are expressed or modified in cellular functions related to homeostasis under stressful conditions (here: posttranslational modifications, apoptosis, cancer). We expect functional similarity of both lacertid and other vertebrate pEAGs to be high, showing functional constraint. 
Lastly, we aim to understand the extent of repeated positive diversifying selection on the same genes in different species and settings. We identify the genes that are repeatedly selected in response to abiotic changes in more than one species and compare the number of such events against simulations to infer functional constraint. Here, we expect to find both functional similarity as well as evidence for constraint in the modification of pEAGs across different taxa.

\section{Material and Methods}

\section{Analysis of positive diversifying selection of genes adapting to latitudinal climate in Lacertidae}

RNAseq data of 24 lacertid taxa were obtained from Garcia-Porta et al. (Garcia-Porta et al., 2019) (collection and ethics permits listed therein). The final alignment contained 6,269 gene sequences. Gene sequences obtained from RNAseq with no missing taxa were further checked for alignment errors including HMMCleaner for false negatives to pre-empt including artefacts in nucleotide-level analyses. Ten sequences were found to contain such potential artifacts and were removed from this data set, resulting in transcriptomes annotated to 5,498 unique gene symbols. A maximum likelihood tree was generated from the concatenated alignment, which is also published in the same study (Garcia-Porta et al., 2019). From this final dataset for 24 lacertids (22 nominal species; two species with two divergent populations at subspecies level each) and one outgroup, we selected a subset of 695 genes with no missing sequences in any of the species to analyze for signatures of positive diversifying selection. The aBSREL (Smith et al., 2015) random-effects branch site model as implemented in HyPhy (Kosakovsky Pond et al., 2005) was used to find genes and lineages subject to episodic positive diversifying selection. The full model was run in exploratory mode for all branches with Holm-Bonferroni correction. Summary statistics of $\mathrm{dN} / \mathrm{dS}(\omega)$ were computed across all genes, and for each branch where episodic positive diversifying selection was identified.

In order to establish a possible relationship between genes found to be under positive diversifying selection and aspects of climate, beyond the exome-wide assessment performed in Garcia-Porta et al. (Garcia-Porta et al., 2019), we tested for correlations between their relative evolutionary rate rho and a 
set of "traits" using the package RERconverge in R (Kowalczyk et al., 2019). Continuous "traits" comprised annual habitat hours above $30^{\circ} \mathrm{C}$, median preferred temperature $\left(\mathrm{T}_{\text {pref, }}\right.$, species medians measured experimentally), median evaporative water loss (IWL, species medians measured experimentally), and the first multidimensional metric scaling variable of osteomorphology (morph1mds1, 89 morphological characters measured with CT-scans). All continuous trait data are available in the Supplementary Materials of Garcia-Porta et al. (Garcia-Porta et al., 2019). A Principal Component Analysis was performed to better understand correlative relationships among the continuous "trait" variables.

Binary "traits" analyzed were sets of species inhabiting hot, warm, or cold environments as test branches against the rest of the phylogeny as reference branches. "Hot"-adapted species whose habitats experience more than 3000 annual hours above $30^{\circ} \mathrm{C}$ were the tropical lizards Takydromus sexlineatus and Holaspis guentheri. "Warm"-adapted species whose habitats have more than 1300 annual hours above $30^{\circ} \mathrm{C}$ were Apathya cappadocica, Algyroides nigropunctatus, Dalmatolacerta oxycephala, Hellenolacerta graeca, Holaspis guentheri, Mesalina olivieri, Phoenicolacerta laevis, Scelarcis perspicillata, Takydromus sexlineatus, and Timon pater. "Cold"-adapted species whose habitats have less than 700 annual hours above $30^{\circ} \mathrm{C}$ were Archaeolacerta bedriagae, Dinarolacerta mosorensis, Iberolacerta bonnali, Lacerta agilis, Podarcis muralis, and Zootoca vivipara.

Signatures of selection in genes measured by $\omega$ can, in addition to positive diversifying selection, also be an outcome of relaxation of prior selective constraint (Lahti et al., 2009; Wertheim et al., 2015), which may lead to misidentification of selective forces at play especially in high $\omega$ genes. We therefore additionally ran RELAX (Wertheim et al., 2015) in HyPhy in full mode, specifying taxa inhabiting warm or cold environments as test branches against the rest of the phylogeny as reference branches. A functional genetic network of all genes found to harbor signatures of selection was then generated with Cytoscape v.3 (Shannon et al., 2003) using the STRING app against the Anolis carolinensis genome as background, the closest related lizard with a sequenced and comprehensively annotated genome. The network was then tested for functional enrichment in Cytoscape using the ClueGO app v.2.5.0 (Bindea et al., 2009), with the CluePedia plugin (Bindea et al., 2013). 


\section{Meta-analysis of pEAGs and their functions across vertebrates}

We compiled a matrix of pEAGs in other vertebrate endotherms and ectotherms, adding the 200 genes under positive diversifying selection here identified for Lacertidae (original studies were identified through searching online publication repositories PubMed and Google Scholar for keywords and are listed in Supplementary Table S1). Support for performing comparative functional genomics comes from the fact that orthologs across taxa are, despite their phylogenetic definition, commonly identified by conserved sequence and function (Altenhoff \& Dessimoz, 2009). In addition, to ensure that genes could be compared across vertebrate taxa, we verified that all identified genes had orthologs across representative vertebrate genomes using OrthoDB (Kriventseva et al., 2019). The STRING database plugin within the Cytoscape (Shannon et al., 2003) software was then used to generate a functional genomic PPI (Protein-Protein Interaction) network from these genes, using human PPI databases (Altenhoff \& Dessimoz, 2009). We recorded and visualized whether single pEAGs (nodes) within this network additionally were identified to have adapted to environmental changes in more than one species, whether they were responsive to stress, and which of the Lacertidae genes under positive diversifying selection were part of this network. Gene functions in the form of Gene Ontologies (GO), for pEAGs that in addition to being repeatedly selected in different species were part of the resulting vertebrate network ( $\mathrm{N}=902$ genes, Supplementary Table S1), were generated and grouped by function in ClueGo within Cytoscape (Bindea et al., 2009). Significance was based on false discovery ratecorrected p-values of overrepresented GOs obtained with ClueGo. We then grouped the resulting GO terms by functional categories for climate adaptation as previously defined (Porcelli et al., 2015; Rodríguez et al., 2017; Wollenberg Valero et al., 2014). GO functions which did not fall into these previously predicted categories were recorded separately. We subsequently repeated this analysis for only those genes under positive diversifying selection in Lacertidae that were additionally part of the functional network (pEAGs, $\mathrm{N}=171$ ), and for those genes that were under positive diversifying selection in Lacertidae but were not part of the functional network (less likely to be EAGs, N=29). 
The 695 genes in our RNAseq alignment of Lacertidae represent a subset of genes of the entire aligned transcriptomes that were selected for analysis of positive diversifying selection based on the absence of paralogs and presence of transcripts in all 24 lacertid taxa, as explained above. Consequently, the 200 genes under positive diversifying selection found within this dataset might represent a non-random subset of the transcriptome dataset consisting of 5,498 annotated genes, with genes related to fundamental metabolic cell processes possibly being overrepresented as they would be more likely found in the transcriptomes of all studied lacertid species. To test to what extent the functional properties of Lacertidae genes under positive diversifying selection are similar to housekeeping genes and whether they are different from a random subset of genes, we generated comparison groups in the form of replicates of 200 randomly drawn gene sets. These were drawn from the total sequenced exomes ( 20 sets), and from the genes not found under positive diversifying selection (20 sets), as well as from 451 human housekeeping genes expressed in different types of healthy cells (brain, kidney, prostate, liver, muscle, lung, vulva, 10 sets, Hsiao et al., 2001). Functional similarity of genes repeatedly selected in more than one species among the set of vertebrate pEAGs was compared against 20 sets of 902 genes randomly drawn from 9,840 genes with identical functions obtained via AmiGO, and genes randomly drawn from the human genome $(20$ sets, obtained via www.molbiotools.com). GO terms are a hierarchical semantic classification method for gene functions (Wang et al., 2007). Functional similarity of gene products can therefore be quantitatively estimated through the semantic similarity of GO terms (Wang et al., 2007). We compared the mean semantic similarity of gene sets to populations of randomized gene sets using $\pm 95 \%$ prediction intervals and Ztests.

We additionally tested whether the number of 480 genes found to be repeatedly positively selected in different vertebrates was higher or lower than if all genes with the same functions had been randomly sampled in the same way. For this purpose, we simulated the number of draws that must be made from the set of all genes with identical functions to the vertebrate pEAGs $(N=9,840)$ to yield the same number (a modified Birthday problem, see Supplementary Methods). We tested for differences between the observed number of genes and the simulated distribution using a Z-test. 
Relationship between of $p E A G s$ and functions with involvement in organismal stress response as well as cellular homeostasis

A range of -omics studies of the organismal stress response as well as other targeted studies of invertebrate and cellular responses to abiotic stressors (cold, heat, hypoxia, desiccation, detoxification hibernation and hypersalinity) were manually compiled and queried for the appearance of pEAGs (a full list of studies can be found in Supplementary Table S2). We acknowledge that such studies are being continually published but have attempted a complete inventory at the time of analysis. For the cellular-level data, we first included involvement of pEAGs in cellular pathways active in cancer. For this purpose, gene expression data for 813 pEAGs were obtained from microarray experiments of 89 cancers (Lukk et al., 2010) and from universal reference tissue. To explore involvement of pEAGs in apoptosis events, "zombie genes" were then identified in our data matrix (Pozhitkov et al., 2017). Data on presence and type of experimentally confirmed PTMs of pEAGs was obtained from the PhosphoSite database (www.phosphosite.org, last accessed February 2019), and counts compared against the previously described 20 replicates of 200 randomly drawn genes from the human genome using MannWhitney U test and Z-statistic of randomized Mann-Whitney W statistic. To investigate differences between ectotherm and endotherm species, pEAGs expressed during embryonic development for zebrafish as an ectotherm model species (White et al., 2017) and humans as an endotherm model species (Yi et al., 2010) were also binary coded. pEAGs were binary coded for their association with predefined candidate functions for environmental adaptation. The resulting gene/function matrix was subsequently analyzed via multiple correspondence analysis (MCA) in R (package FactoMineR, Lê et al., 2008) to align genes into functional clusters. Statistical support for MCA dimension groupings were obtained from 100,000 multiscale bootstrap replicates in the R package pvclust (Suzuki \& Shimodaira, 2006). For further details, see Supplementary Methods. 

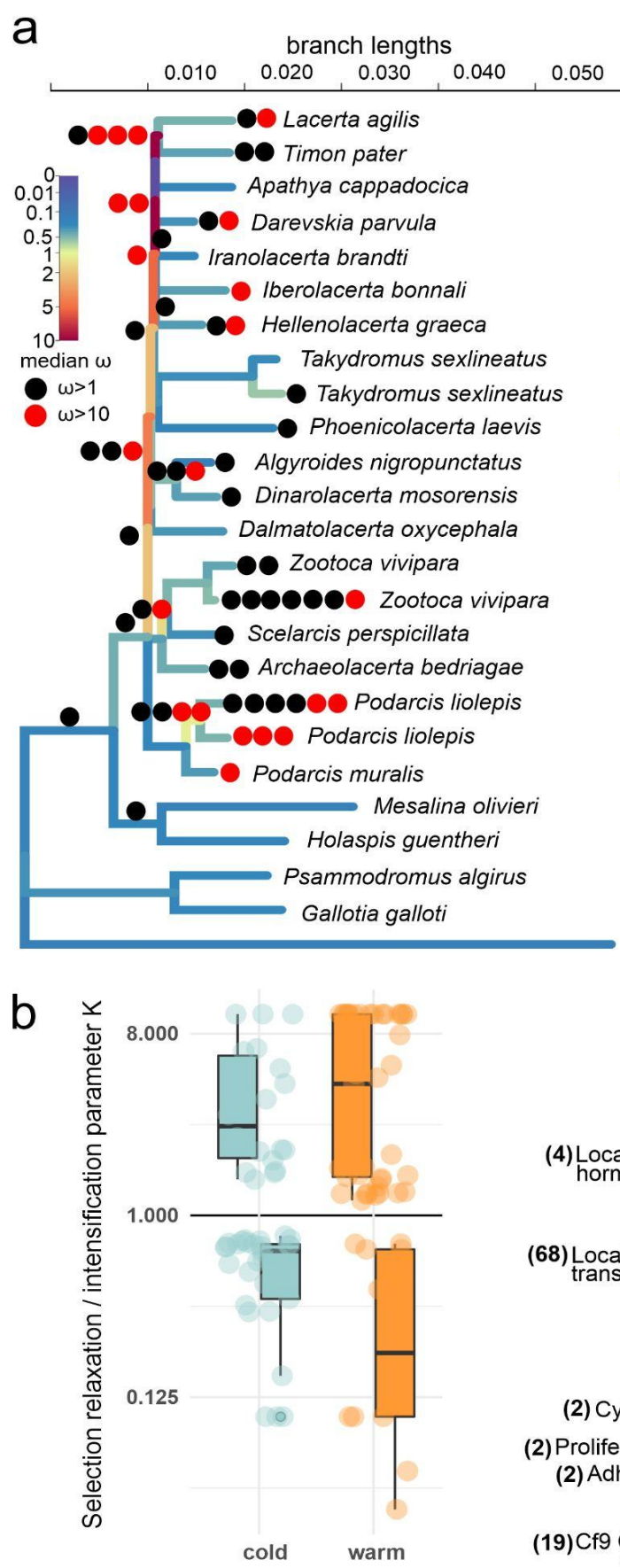

Habitat type

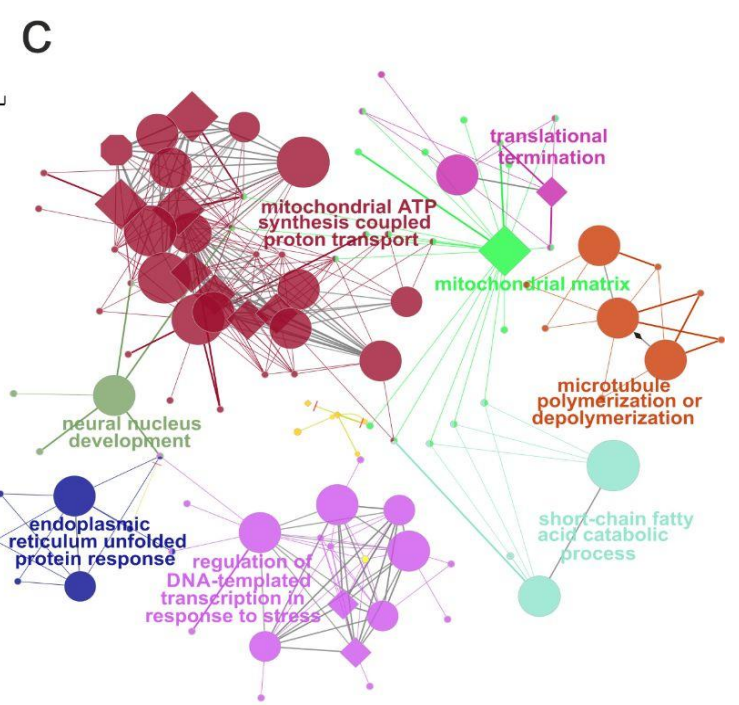

Blanus cinereus

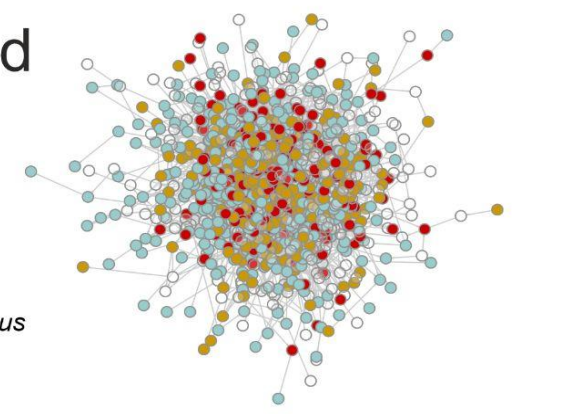

e

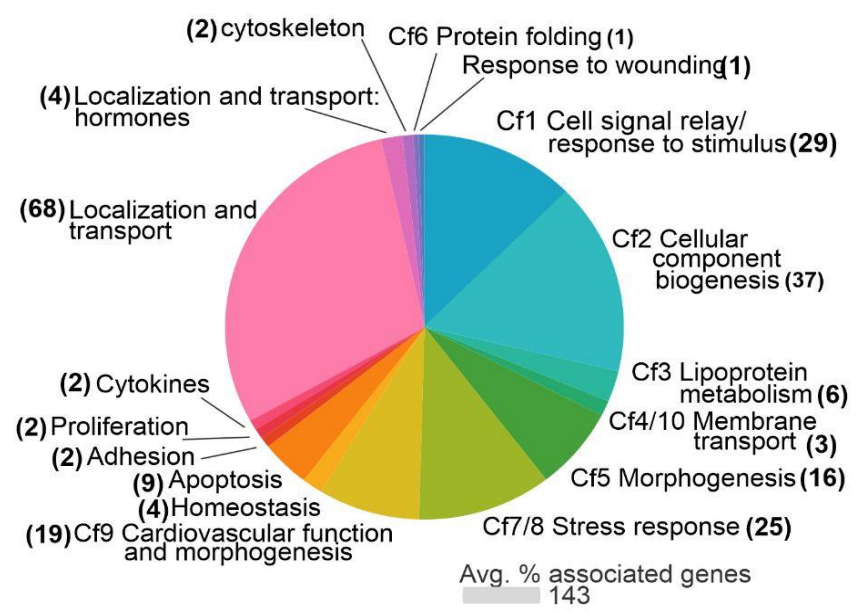

Fig. 1. Phylogenetic and functional properties of lacertid and vertebrate pEAGs. (a) Visualization of median selection coefficient $(\omega)$ on an RNAseq maximum likelihood phylogram of 24 lacertid species for 200 genes under episodic positive diversifying selection. Genes with $10>\omega>1$ and $\omega>10$ are shown along the branches as circles. (b) Box and jitter plots showing genes with significant evidence for relaxation $(\mathrm{K} \leq 1)$ and intensification $(\mathrm{K} \geq 1)$ of selective pressure among cold and warm-adapted clades. (c) Network of significantly enriched functions for these genes in Lacertidae (circles - biological process; diamond - molecular function; octagon - cellular component; size of circles - number of genes per category). (d) Functional genomic network containing 902 pEAGs across endotherm and ectotherm vertebrates (blue -genes that adapted to environmental parameters more than once in different species; ochre-genes that are stress responsive, maroon -genes that adapted in more than one species and are stress responsive). (e) Graph of functional categories of significantly overrepresented ClueGo Gene Ontology groups, for the $902 \mathrm{pEAGs,} \mathrm{categorized} \mathrm{by} \mathrm{candidate} \mathrm{functions} \mathrm{(Cf)} \mathrm{and} \mathrm{additional} \mathrm{functions.}$ Numbers in labels refer to numbers of Gene Ontology terms within each category. 


\section{Results}

\section{Genes under episodic positive diversifying selection in Lacertidae}

Among 5,498 ortholog genes compiled from the transcriptomes of 24 lacertid taxa (Garcia-Porta et al., 2019), we identified 200 genes to have evolved under episodic positive diversifying selection. The estimated median $\omega(\mathrm{dN} / \mathrm{dS}$ ratio) per branch for these 200 genes was plotted onto a maximum likelihood RNAseq phylogeny (Figure 1a). Branches splitting from basal nodes, except Podarcis, showed the highest median values of $\omega$. The majority of $10>\omega>1$ genes were located on the (long) terminal branches. In contrast, $\omega \geq 10$ genes were concentrated both along the phylogeny backbone and on specific terminal branches. To assess whether genes with high $\omega$ values could, instead of being a result of positive selection, alternatively have resulted from relaxation of prior selection, we performed relaxation tests between phylogenetic models of the selection relaxation/intensification parameter $\mathrm{K}$ for species adapted to warm and cold environments (Wertheim et al., 2015). We found evidence for selection relaxation in 47 genes, and evidence for selection intensification in 18 genes in response to cold environments. We also found ten genes with evidence for selection relaxation and evidence for selection intensification in 45 genes in response to warm environments (Figure 1b, Supplementary Table S3). However, only two of these genes had both evidence for (weak) relaxation of selection in cold environments, combined with a $\omega$ value of more than 1.4 (gene RBM5 with $\mathrm{K}=0.763$, $\mathrm{LR}=4.286$, $\mathrm{p}=0.038$ and $\omega=5.09$; and gene NPEPPS with $\mathrm{K}=0.703, \mathrm{LR}=5.406, \mathrm{p}=0.02$ and $\omega=10$ ). NPEPPS also was the only gene with evidence for both strong selection intensification in response to warm environments combined with high $\omega(\mathrm{K}=10, \mathrm{LR}=8.316, \mathrm{p}=0.004$ and $\omega=10)$. The remaining genes with evidence for either relaxation or intensification of selection all had $\omega \leq 1.4$, see Supplementary Table S3.

To test whether the 200 genes identified to be under positive diversifying selection were adapting to climate in Lacertidae, we tested whether they convergently increased or decreased their relative evolutionary rates rho in response to different habitat and physiological characteristics across different branches of the phylogeny. We identified sets of genes which showed significant rate accelerations in "hot" $(\mathrm{N}=22)$, "warm" $(\mathrm{N}=21)$, and "cold" $(\mathrm{N}=12)$-adapted species (Figure 2a-c). Overall, there were 
similar amounts of genes with rates being associated positively vs. negatively to the respective habitat types. With regards to continuous characters, we found a positive but non-significant correlation between the evolutionary rate correlations of annual hours above $30^{\circ} \mathrm{C}$ and $\mathrm{T}_{\text {pref }}$ (Figure $2 \mathrm{~d}$, Kendall's rank correlation tau $=0.08, \mathrm{z}=1.733, \mathrm{p}=0.083$ ). We found significant negative correlations between the relative evolutionary rates of annual hours above $30^{\circ} \mathrm{C}$ and (i) evaporative water loss (Figure 2e, iwl, tau $=-0.305, \mathrm{z}=-6.525, \mathrm{p}=6.793 \mathrm{e}^{-11}$ ) and (ii) general osteomorphology (Figure 2f, morph1mds1, tau $\left.=-0.243, \mathrm{z}=-5.202, \mathrm{p}=1.969 \mathrm{e}^{-07}\right)$. Principal Component Analysis revealed that three of the four continuous "trait" predictors (annual hours above $30^{\circ} \mathrm{C}, \mathrm{T}_{\text {pref, }}$ and morphology) were correlated and formed a Principal Component explaining $48.5 \%$ of total variance. IWL comprised the second Principal Component explaining $29.2 \%$ of total variance (in total, $77.7 \%$ explained by both Principal Components). We therefore explored how the relative evolutionary rates of this temperature-associated first Principal Component differed between genes under positive selection vs. not under selection. We found that genes under selection had significantly lower median relative evolutionary rates than genes not under selection (Figure 3a, Mann-Whitney U test MW-U $=2901, \mathrm{p}<0.0001$ ), indicating that overall, genes under selection are under higher constraint than genes not under selection.
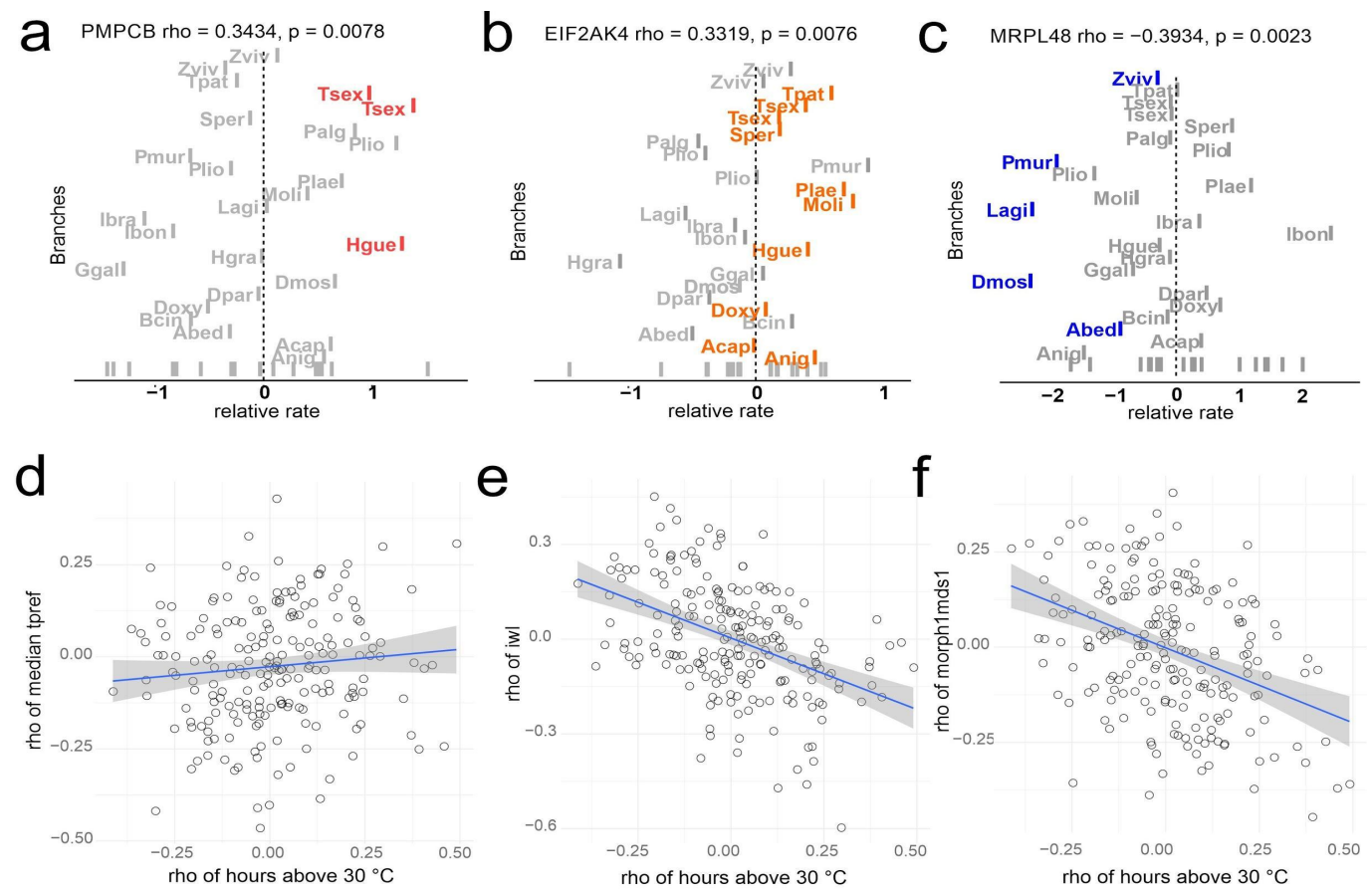

Fig. 2. Association of climate with evolutionary rates and convergence in genes under positive 
diversifying selection in Lacertidae. (a-c) show genes with highest association between relative evolutionary rate (rho) and habitat types (hot, warm, and cold). The gene PMPCB (Mitochondrial processing peptidase beta subunit) shows accelerated evolutionary rate in taxa adapted to hot habitats, indicating positive selection (a). The gene EIF2AK4 (Eukaryotic Translation Initiation Factor 2 Alpha Kinase 4) shows accelerated evolutionary rate in taxa adapted to warm habitats, indicating positive selection (b). The gene MRPL48 (Mitochondrial ribosomal protein L48) shows decelerated evolutionary rate in taxa inhabiting cool habitats, indicating purifying selection and increased evolutionary constraint (c). Tick marks at the base of plots (a-c) represent untested interior nodes of the tree. Lacertid species are abbreviated with first initial of genus and first three initials of species. Across all 200 genes found under positive diversifying selection in the lacertid phylogeny, the association parameter rho of relative evolutionary rate with habitat hours above $30^{\circ} \mathrm{C}$ was (d) only weak positively correlated with the rho of median preferred temperature (Kendall's rank correlation tau $=0.08, \mathrm{z}=1.733, \mathrm{p}=0.083$ ), indicating positive selection for high $\mathrm{T}_{\text {pref }}$ in warm habitats. In contrast, (e) the rho of habitat hours above $30^{\circ} \mathrm{C}$ was significantly negatively correlated with the rho of evaporative water loss (iwl, tau $=-0.305, \mathrm{z}=-6.525, \mathrm{p}=6.793 \mathrm{e}^{-11}$ ), and likewise (f) to general morphology (morph1mds1, tau $=-0.243, \mathrm{z}=-5.202, \mathrm{p}=1.969 \mathrm{e}^{-07}$ ), indicating positive selection of IWL and general morphology in cooler habitats.

Based on literature meta-analyses and experimental confirmation of genes adapting to climate, a set of candidate functions related to climate adaptation had previously been defined in vitro and in silico, and verified in natural populations (Porcelli et al., 2015; Rodríguez et al., 2017; Wollenberg Valero et al., 2014). We tested whether newly identified and compiled pEAGs were enriched in these functions. The lacertid genes were significantly enriched (Figure 1c) in several previously defined candidate functions potentially related to climate adaptation (Porcelli et al., 2015; Rodríguez et al., 2017; Wollenberg Valero et al., 2014), and in cytoskeletal processes as additional, not predicted functional category (Tables S4-S5 showing allocations of candidate functions and GOs enriched with $\mathrm{p}<0.05$ in the gene set per candidate function).

Functional analysis of vertebrate $p E A G s$ and their repeated selection in different species for abiotic environmental adaptation

1,100 pEAGs across ectotherm and endotherm vertebrates were compiled from -omics studies of adaptation to different or novel abiotic environments. These genes resulted from statistical comparisons of evolutionary lineages such as we have done here for the Lacertidae dataset. Including the 200 Lacertidae genes, the set of 1,100 genes were involved in adaptation to different environmental conditions comprised of thermal differences, latitudinal climate, altitude or season, sulfidic water in streams, osmotic differences, desiccation, hypoxia, or wet-dry environmental gradients (see Table S1). To ensure that these genes were comparable across these different species, we manually verified that all 1,100 pEAGs were orthologs in Vertebrata with OrthoDB (Kriventseva et al., 2019) which enabled 
these cross-species comparisons (doi:10.6084/m9.figshare.15010887). Of the 1,100 pEAGs, 902 or $83 \%$ were part of a large PPI network characterized by short path lengths and consisting of only one connected component (Figure 1d). Among these, 480 genes or $43.6 \%$ were repeatedly positively selected in response to environmental adaptation in multiple, not closely related vertebrate clades (Figure 1d, Table S6). The Gene Ontology (GO) terms for this network covered all except one a priori predicted function potentially related to climate adaptation, which was muscle use and -development (Figure 1e, Table S4, S7). Additional significantly enriched GO terms within this network were related to the cytoskeleton, apoptosis, and localization and transport (Table S4, S7, Figure 1e). The latter function encompassed 68 related GO terms and 459 genes (45\%). $86 \%$ or 171 of the 200 selected genes in Lacertidae were also part of this functional network (Figure S1, Table S4). We consider it likely that at least these 902 genes (171 of Lacertidae) connected to each other by climate adaptation-relevant functions and by evidence for repeated positive diversifying selection represent true EAGs, while this is less likely for the 29 Lacertidae genes not connected to this network.

Fifty of the Lacertidae genes found to be under selection were shared by more than one (between two and ten each) terminal taxa in the lacertid phylogeny. Five of these contained an RNA recognition motif (TIAL1, U2SURP, ZCRB1, NCL; STRING-determined PPI enrichment p-value $=0.0506$ with Anolis carolinensis as background). Fifteen genes were under selection in both Lacertidae and other vertebrates including endotherms. Among these, two genes were enriched in Rad60/SUMO like domain (HIPK2, UBA2), and two were ribosomal proteins (MRPL15, MRPS31, STRING-determined PPI enrichment p-value $=0.014$ with Anolis carolinensis as background). We tested whether the number of 480 repeatedly selected genes out of 1,843 genes drawn from all datasets in this study (accounting for some of the 1,100 individual genes being repeatedly selected, sometimes in more than two species), was higher than expected under regular gene functional constraint. In 100,000 simulations, we found that to obtain an identical number of more than once repeatedly selected genes solely by chance, on average 3,447 ( \pm 63.8) genes would have to be drawn from the set of 9,840 genes with identical GO terms to obtain 480 genes at least twice. This is significantly higher than the empirical number of draws performed in this study $(1,843$ draws, Figure $3 b, Z=-2493, p \leq 0.00001)$. This shows that the high percentage of $43.6 \%$ repeatedly selected genes across vertebrate climate adaptation events is an 
outcome of functional constraint that is higher than if genes with the same function were randomly selected at least twice from the genes with similar functions in response to abiotic selection.

When lacertid pEAGs were separately subjected to GO enrichment analysis, two additional previously defined candidate functions for climate adaptation were recovered (cell signalling and muscle-related processes; Table S4, S5). For the 29 Lacertidae genes under positive diversifying selection that were not part of the network (and less likely constituting true EAGs), no functional GO term enrichment could be detected (Table S4). Lacertid pEAGs also had higher semantic similarity of GO terms than those 29 not part of the network, and higher semantic similarity than randomized replicates of all other comparison groups ( 29 genes not under positive diversifying selection $Z=2.5, p$ $=0.012$, all other annotated orthologs in transcriptomes $\mathrm{Z}=5, \mathrm{p} \leq 0.00001$, randomly drawn genes $\mathrm{Z}$ $=3.6, p=0.0003$, Figure 3c), except human "housekeeping" genes. (Figure $3 c, Z=1, p=0.159$ ).

a

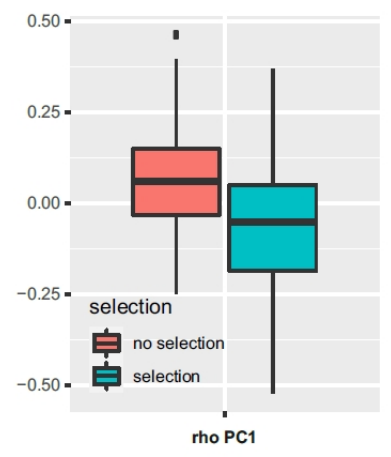

b

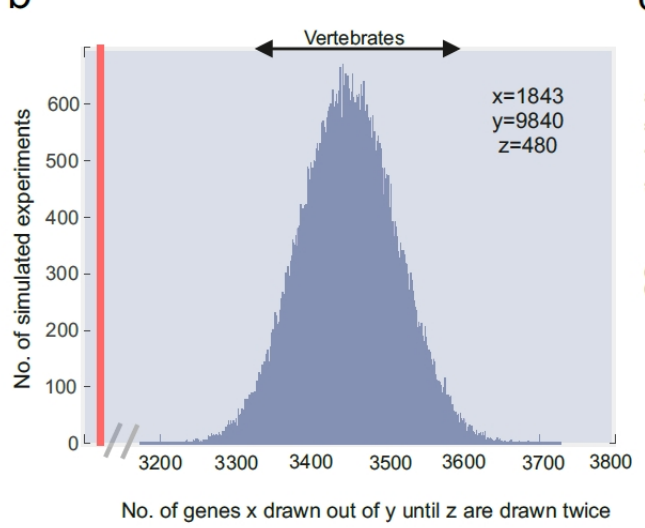

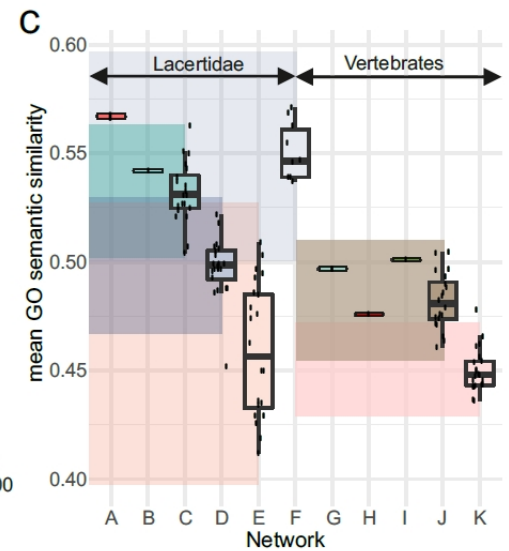

Fig. 3. Comparison of lacertid genes under positive diversifying selection and vertebrate pEAG. (a) Box plot showing relative evolutionary rates $r$ ho of the first Principal Component of temperature-associated variables (habitat hours above $30^{\circ} \mathrm{C}$, median $\mathrm{T}_{\text {pref, }}$ and general morphology, $\mathrm{PC} 1$ ) for genes under positive diversifying selection being significantly lower than those not under selection in Lacertidae $\left({ }^{* * *}\right)$. This indicates constraint in evolutionary rates of genes under selection. (b) Number of genes that was drawn in this study $(1,843)$ to find 480 repeatedly selected genes (red bar) is significantly lower than the number of genes obtained in $N=100,000$ simulations of random draws from a set of 9,840 genes with identical GOs to draw the same 480 genes at least twice (histogram, $* * *$ ). (c) Comparisons of functional (semantic) similarity of GO terms between genes under positive diversifying selection in Lacertidae and comparison gene sets (A-F), and between vertebrate pEAGs and comparison gene sets (G-K). Boxes show medians, 25-75 quartiles, and jittered raw data. Transparent bands indicate modelled $\pm 95 \%$ prediction intervals. The plot shows that the 200 lacertid genes under positive diversifying selection (A) have the highest functional similarity. Their semantic similarity is higher than that of 29 genes under positive diversifying selection not part of the network (B), of 20 random subsets of 200 genes drawn from the 495 genes not under positive diversifying selection $(\mathrm{C}, * *)$; of 20 random subsets of 200 genes drawn from 5,498 annotated ortholog genes with incomplete taxon sampling obtained from RNAseq (D, $* * *)$, of 20 replicates of 200 random human genes $(\mathrm{E}, * * *)$, but not higher than 10 replicates of 451 human housekeeping genes (F) 902 vertebrate pEAGs of the vertebrate functional genomic network (G) split up into repeatedly selected (H) and not repeatedly selected (I) gene sets that overlap with the $95 \%$ prediction intervals of 20 replicates of 902 random subsets of genes with identical GOs (J). Gene sets G and J significantly differ in Z-tests (***). Vertebrate pEAGs, however, have significantly higher functional similarity than 20 random subsets of 902 random vertebrate 
genes $(\mathrm{K}, * * *)$. p-values are denoted as $* \leq 0.05 ; * * \leq 0.01 ; * * * \leq 0.001$.

We next tested whether the functions of vertebrate pEAGs were more similar than different comparison gene sets. The vertebrate pEAG network had significantly more interactions between the genes than expected if they were randomly drawn from the genome, with a PPI enrichment p-value of $2 \mathrm{e}^{-13}$. It also had significantly higher functional similarity than random networks of comparable size (Figure $4, Z=137.69, p=0.00001$ ). Its functional similarity was, however, within the $95 \%$ prediction intervals of randomized networks belonging to genes with the same GO terms, but still was significantly more similar than genes belonging to these same $\mathrm{GO}$ terms $(\mathrm{Z}=34.37, \mathrm{p}=0.00001)$.

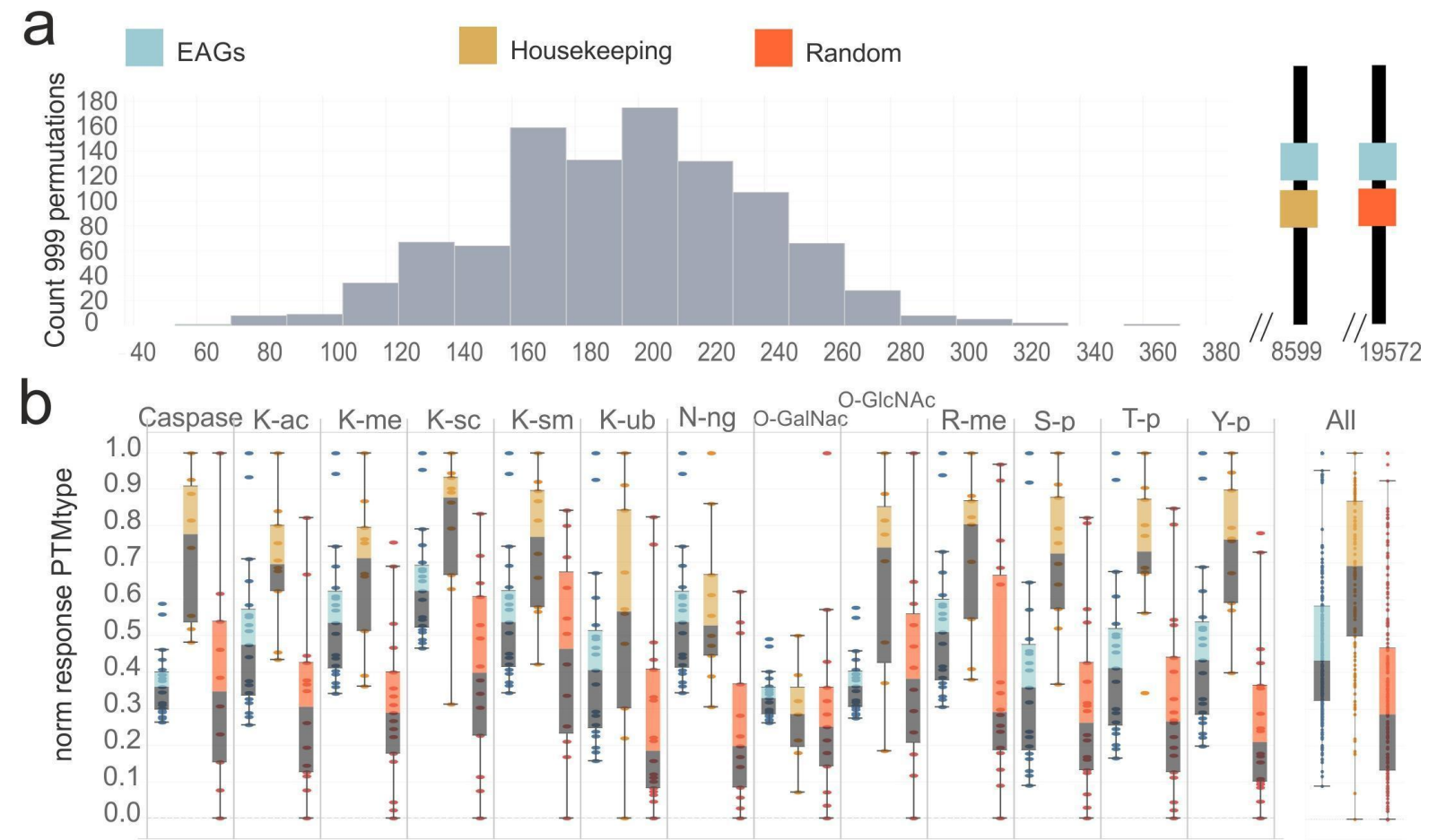

Figure 4. PTMs of proteins encoded by pEAGs and random gene lists. (a) A randomized distribution of Mann-Whitney W statistic across random and environmental adaptation PTM values $(\mathrm{N}=520)$, obtained by 999 permutations, yielded a maximal W-value of 365 and shows that the boundaries of this distribution do not overlap with the test W-values of either pEAGs against housekeeping genes or pEAGs against randomly drawn genes (black bars). PTM data obtained for humans were first normalized by PTM type. PTM counts were then compared by (b) type between 20 random gene sets, 20 random subsets of pEAGs, and 20 random subsets of human housekeeping genes. Overall, pEAGs encoded proteins with significantly more PTMs than random genes and less PTMs than housekeeping genes (see right plot labelled "ALL").

\section{Evidence for involvement in different cellular functions}

We subsequently tested whether all pEAGs, and repeatedly selected pEAGs, have common 
characteristics with respect to by (i) a priori defined candidate functions for climate adaptation, (ii) relatedness to the organismal stress response, (iii) aspects of cellular homeostasis (cancer and apoptosis), and (iv) enrichment in specific post-translational modifications (PTMs).

One hundred ninety-eight pEAGs or 18\% had "stress response" as a significant GO term (Table S6). Furthermore, we found empirical evidence in the literature (not restricted to vertebrates) for 329 (29.9\%) genes being differentially expressed in response to abiotic environmental stress (including cold stress, heat stress, hypoxia stress, and others including hibernation, desiccation and detoxification, Table S6, the complete data is provided in doi:10.6084/m9.figshare.15010887). Of these, $125(38.0 \%)$ were upregulated and $74(22.5 \%)$ were downregulated (Figure S2). The top three candidate functions for climate adaptation represented by these genes were "cellular component biogenesis", "morphogenesis", and "response to oxidative stress / stress response (incl. thermal stress)" (Table S8S9). All these genes were part of the functional network. Several of them were heat shock proteins; however, the two most recorded genes responding to all analyzed aspects of stress response across different species were the less-well studied genes BAG3 and AHSG. AHSG furthermore responded to all four groups of stressors, together with PKLR and SQOR (Figure S3a).

Concerning cellular pathways active in cancer as possible pEAG candidate function, we found that pEAGs used by selection in more than one species have lower expression values in both cancer and healthy tissues, compared to genes only selected with respect to environmental changes in one species (Figure S3b), whilst ratios of pEAG gene expression between cancer and reference conditions did not differ (Table S10). Five genes (COL3A1, NCAN, EDNRB, SLC6A1, FCGBP and SYT11) of the data set however, were involved in more than 80 cancer types (Figure S4, Table S11).

With respect to PTMs, evidence for human gene product PTMs was present in $19 \%$ of pEAGs, and $21 \%$ genes furthermore had the function "gene regulation - protein modification" (Table S2). The gene products of pEAGs overall had significantly more PTMs than randomly drawn genes (Table S2, MannWhitney $\mathrm{W}=19572, \mathrm{p} \leq 2.2 \mathrm{e}^{-16}$ ), and significantly less PTMs than randomly drawn human housekeeping genes $\left(\mathrm{W}=8599, \mathrm{p}=2.57 \mathrm{e}^{-15}\right)$. These results remain significant after comparison with a randomized distribution of the $\mathrm{W}$ statistic (pEAG to random genes $\mathrm{Z}=443.726, \mathrm{p} \leq 0.001$; $\mathrm{pEAG}$ to housekeeping genes $Z=192.44, p \leq 0.001$, Figure 4). 
To visually align pEAGs to candidate functions as well as other cellular functions (ii-iv mentioned above), six functional dimensions of pEAGs were extracted by Multiple Correspondence Analysis. Figure 5 shows the dendrogram obtained by pvclust following 100,000 bootstrap replicates. Eleven hierarchical clusters were supported with bootstrap values $>95 \%$. Other than expected, genes were not partitioned into clusters that could be identified as clearly ectothermic or endothermic (for more details see Supplementary Results). Instead, genes were clustered by function and various other evolutionary aspects.

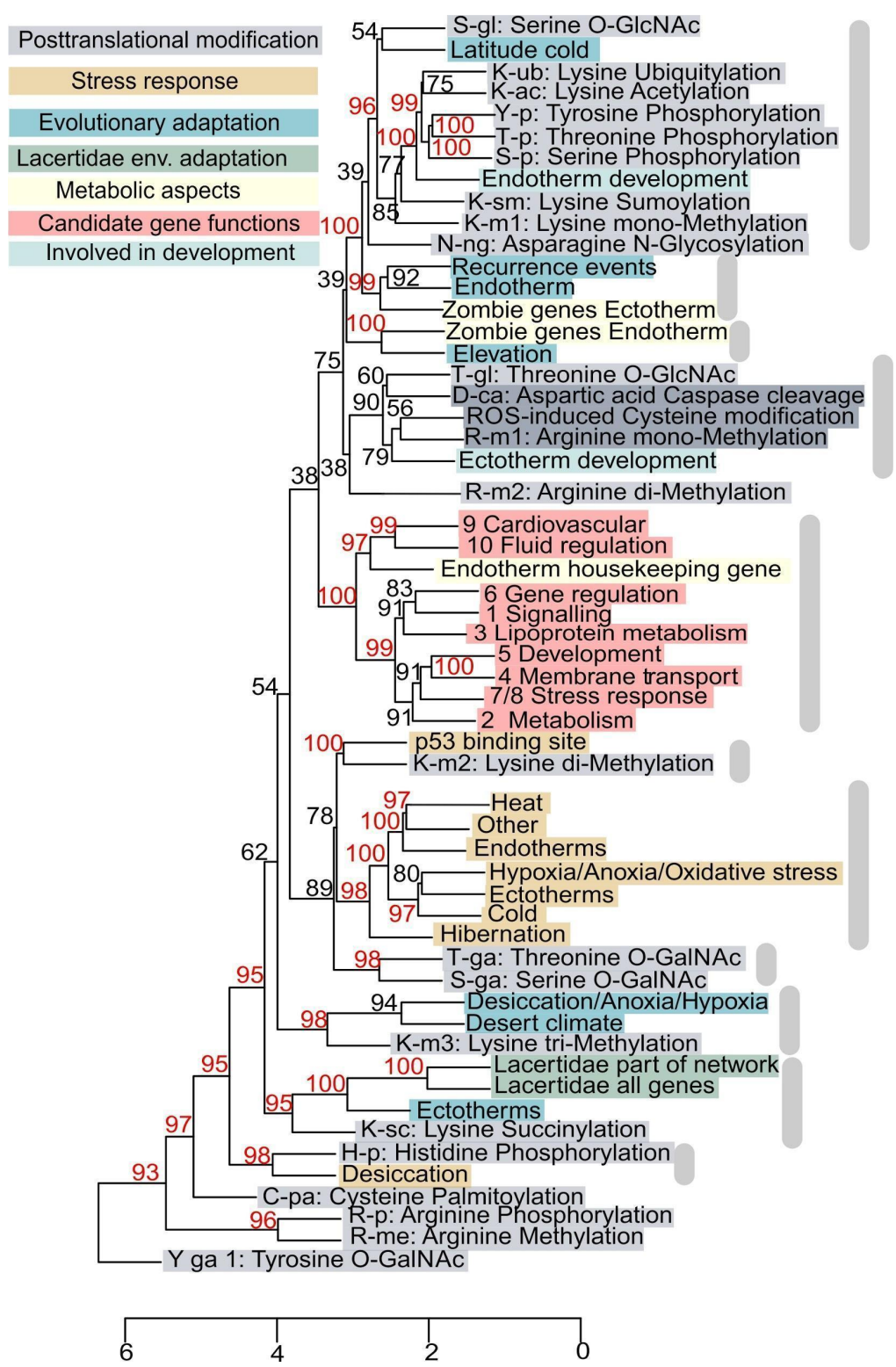

Fig. 5. Results of Multiple Correspondence Analysis (MCA), represented as a dendrogram, to scale pEAGs by different organismal and cellular functional and evolutionary aspects. Statistical support was obtained via multiscale bootstrap (N=100,000 replicates; Suzuki \& Shimodaira, 2013). Recurrence 
events - events where the same gene is under repeated environmental selection in two or more species. 11 functional clusters were obtained with combined bootstrap support (alpha $>0.9$, red numbers on nodes), and standard error of estimate $<0.01$ (not shown).

\section{Discussion}

In this contribution, we investigated whether the genomic basis of environmental adaptation in lacertid lizards and other vertebrates is characterized by specific functions and gene subsets, and whether these genes play a part in the response to stress both experienced at the organismal level (abiotic environmental stress) and at the cellular level (PTMs, activation in cancer, embryonic development or during apoptosis "Zombie genes"). We here found evidence for higher than random levels of identity and functional similarity among genes that have adapted to abiotic selective regimes among different vertebrate taxa and physiological strategies, as well as involvement in the stress response.

We found 200 genes that show signatures of positive diversifying selection in Lacertidae, a group of lizards inhabiting a wide climatic gradient. Taxa that inhabit hot or cold areas for a long time may experience relaxation of selective pressure in genes first used to adapt to such environments (Lahti et al., 2009). Conversely, selection in genes which supported heat adaptation in ancestral branches could be relaxed if a clade expands northwards into cooler climate and selection pressure is shifted to genes that predominantly promote cold adaptation (Lahti et al., 2009). In Lacertidae, we found evidence for strong relaxation in selective pressure with respect to cold environments combined with high $\omega$ in only two genes, NPEPPS and RBM5. However, the large $\omega$ value in NPEPPS was identified only in Podarcis liolepis, a species which was neither a member of the cold-adapted nor warm-adapted comparison groups, and it is still feasible to conclude that the high $\omega$ in P. liolepis was due to an episodic event of positive diversifying selection along its branch. In contrast, it is possible that RBM5 experienced a period of relaxation of selective pressure in correlation with the expansion into colder climate, as its only high $\omega$ value is located on an interior branch separating Timon pater (warm adapted) and Lacerta agilis (cold adapted). A range of other genes had smaller $\omega$ values combined with $\mathrm{K}$ values closer to 1 , which may indicate a more complicated interplay between episodes of positive diversifying selection and relaxation. Overall, an interesting pattern emerges showing that lacertids in warm environments 
experience an overall intensification of selection, and lacertids diversifying into cooler climates a relaxation of selection pressure. This pattern is consistent with prior predictions that selection is stronger in response to heat than in response to cold, due to the asymmetry of the thermal fitness curve (Martin \& Huey, 2008).

Nonetheless, we here found evidence for evolutionary rates of genes with signatures of selection being correlated with physiological adaptations to abiotic parameters. A previous study using the same lacertid dataset with species as data points (Garcia-Porta et al., 2019), found that species with more yearly hours above $30^{\circ} \mathrm{C}$ have higher evolutionary rates, higher preferred temperature, and lower evaporative water loss. With respect to correlations of these predictors amongst each other at the species level, previous phylogenetic linear and second order polynomial regression models (PRM) did not find any of the other predictors to be correlated (cf. Supplementary Table S6 in Garcia-Porta et al., 2019). Results from this current work overall agree with such climate-mediated evolution of phenotypes at the gene level, as relative evolutionary rates of the 200 genes found to be under selection were marginally higher in species with both more habitat hours above $30^{\circ} \mathrm{C}$ and higher median preferred temperature. This indicates positive selection for high $\mathrm{T}_{\text {pref }}$ in warm habitats. Aligned to Garcia-Porta et al. (GarciaPorta et al., 2019), evolutionary rates of these genes were also negatively correlated between habitat hours above $30^{\circ} \mathrm{C}$ and evaporative water loss. In addition, we observed a significant negative correlation of the evolutionary rate correlations of genes of habitat hours above $30^{\circ} \mathrm{C}$ and of osteomorphology. These results indicate positive selection of evaporative water loss and bone morphology in cooler habitats. Two genes under selection accelerating evolutionary rates in clades inhabiting hot and warm habitats, respectively, were PMPCB (Peptidase, mitochondrial processing subunit beta) and EIF2AK4 (Eukaryotic translation initiation factor 2 alpha kinase 4) - these genes could be associated with key innovations enabling adaptation to such habitats. One gene under selection significantly slowing down its rate of evolution in clades inhabiting cold areas is MRPL48 (mitochondrial ribosomal protein L48), indicating the presence of purifying selection or constraint (following interpretations in Kowalczyk et al., 2020).

pEAGs of different species form a tight network with more functional connections among the genes than expected by chance. Across the different species and environmental factors considered, almost 
half of all genes in this functional network (43.6\%) were repeatedly selected in different species in response to changes in the abiotic environment. This percentage was significantly higher than if genes had been randomly recruited from the same functions. For example, 16 pEAGs of Lacertidae under selection in response to a climatic gradient (in both latitude and elevation) have been independently recruited for environmental adaptation in other vertebrates. Other ectotherms use these same genes to adapt to desiccation and anoxia (killifish Austrofundulus limnaeus Wagner et al., 2018), altitude (Himalayan Nanorana frogs, Himalayan Phrynocephalus lizards, Sun et al., 2018). Some endotherm species use the same genes to adapt to latitudinal climate (Yakutian horse, woolly mammoth, minke whale, polar bear, Yudin et al., 2017), deserts (camel and dromedary, Wu et al., 2014), and altitude (Himalayan marmot, Bai et al., 2018). Different than expected, aspects of environmental adaptation and stress response were not clearly separated between vertebrate endotherms and ectotherms, which indicates that the genomic mechanisms and functions of adaptation to abiotic parameters seem to be constrained even among these two, very different physiological strategies. This constitutes another indicator for the presence of genomic constraint in environmental adaptation. For example, Lacertidae adapted to a temperature gradient shared in total five genes under positive diversifying selection with the extinct woolly mammoth adapted to Arctic environments (ETFA, MRPS31, PFAS, PHKB, and SENP5).

In lacertid lizards, terminal branches with very high values of episodic positive diversifying selection were identified mainly in species inhabiting cooler, more seasonal and partially montane environments such as Podarcis muralis and Zootoca vivipara. In contrast, they were absent in hot-arid adapted species such as Mesalina olivieri and species living in tropical environments (Takydromus sexlineatus, Holaspis guentheri).

Adaptation in genes linked to mitochondrial cell respiration and oxidative stress response (proteasome component genes and the chaperone HSP90B1), could facilitate adaptation to cold environments via influencing the metabolic rate (Sokolova, 2018; White et al., 2012). This corroborates previous assumptions that due to biochemical and cell physiological limitations at high temperatures, lower preferred temperatures are more likely to evolve than higher thermal preferences in lizards (Araújo et al., 2013; Munoz et al., 2014). In Lacertidae, genes under positive diversifying selection but 
not part of the vertebrate functional network of pEAGs did not show any functional enrichment and could have evolved in response to positive diversifying selection pressures other than abiotic climaterelated factors. Our comparative genomic approach thus may help disentangle environmental adaptation from covarying factors such as locomotor performance or reproduction (Bourgeois et al., 2019).

The results concerning gene function are in alignment with the hypothesis of evolutionary constraint as well. First, genes under selection in Lacertidae had overall lower relative rates of evolution with regards to changes in habitat temperature, preferred temperature and morphology than genes not under selection. This seems counterintuitive at first but matches results of a previous study which found that increased relative longevity in mammals is also catalyzed in the majority by genes with higher levels of constraint (Kowalczyk et al., 2020). Increased constraint of many genes can be interpreted as traitenabling whereby certain existing biological pathways become increasingly important after new traits have evolved (Kowalczyk et al., 2020). In contrast, a relatively lower number of genes may be involved in catalyzing key innovations, such as the genes we identified to be under positive selection and having faster relative evolutionary rates in response to hot and cold climates. In agreement with this hypothesis, high functional connectedness and comparatively higher values of GO semantic similarity of the pEAGs analyzed in our study indicated that they were indeed drawn from a non-random set of very similar functions (Yu et al., 2010). Many pEAGs fell within predicted candidate functions for climate adaptation which had been proposed for species across the tree of life, independently for Metazoans (Porcelli et al., 2015) and ectothermic vertebrates and invertebrates (Wollenberg Valero et al., 2014), and that had been previously verified in elevational adaptation of Anolis lizard populations (Rodríguez et al., 2017). Due to their universal nature, these functions are quite general which may predispose them for higher similarity in cross-taxon comparisons, but further studies may be able to narrow them down for specific taxonomic groups and type of abiotic selection. We can show that functional enrichment of genes under selection in these candidate functions was not just due to their generality, as we found less semantic similarity in groups of genes randomly drawn from the genome, which would be expected to have similarly general functions. Besides these predefined functions, involvement in cytoskeletal and apoptotic processes, as well as localization and transport emerged as novel environmental adaptation- 
related functions. Both apoptosis and transport-related genes have previously been associated with death-related processes, during which transport genes may become activated attempting to restore homeostasis (Pozhitkov et al., 2017). The cytoskeleton is remodelled through stress and involved in HSP phosphorylation (Dalle-Donne et al., 2001) which in turn protects cytoskeletal integrity (Klose \& Robertson, 2004). Stress furthermore induces changes in cytoskeleton-regulated cell volume (Pedersen et al., 2001).

PTMs have previously been related to regulation of torpor as an environment-induced metabolic process (Morin \& Storey, 2009). Surprisingly, we found that specific PTMs were present in almost all functional clusters associated with environmental adaptation. Genes that were involved in climate adaptation were enriched in various types of PTMs such as serine O-GlcNAc (serine- $\beta$-linked Nacetylglucosamine modification) which are associated with transcription, metabolism, apoptosis, organelle biogenesis, and transport and disease including metabolic syndrome and cancer (Bond \& Hanover, 2015). Moreover, $89 \%$ of all pEAGs were involved in at least one human cancer, and a subset of which had cancer involvement as predominant function - e.g. FCGBP and SYT11, which also adapt to latitudinal climate in polar bear and woolly mammoth (Yudin et al., 2017). This reinforces the previously suggested relationship between abiotic environmental adaptation and homeostasis disturbance via disease (Pozhitkov et al., 2017). Many such "Zombie genes" repeatedly adapted to altitude. These genes are both activated in dying cells, and involve localization and transport processes, which supports these functions as emerging cellular candidate processes for environmental adaptation. Adaptation to hypoxia and desiccation is furthermore associated with the PTM lysine trimethylation (Niu et al., 2015). Lastly, lysine succinylation was linked to genes involved in environmental adaptation in lacertids and other ectotherms, possibly related to UV radiation tolerance (Xu et al., 2016).

The subset of orthologs with presence in all lacertid species could be biased for genes with high expression (that are more likely recovered from all species in a transcriptome-based alignment), and for genes needed for regular cellular functions. Adaptation to the abiotic environment is also likely mediated through genomic elements other than protein-coding genes or differences in gene expression. We therefore likely only analysed a subset of the "true EAG" landscape in lacertid genomes. Evidence from lacertid pEAGs being functionally similar due to abiotic environmental selection, and not just 
because they were a subset based on common properties of expression, comes from their significantly higher functional similarity compared to not selected genes. Secondly, they were functionally more similar to each other than the 5,498 ortholog genes which were missing transcripts for one or more lacertid species and could thus not be analysed for selection - but which also came from the same population of transcripts.

Compared across all vertebrates, pEAGs had higher expression values, higher numbers of PTM sites, and higher functional similarity than other partitions of the transcriptomes, but random sets of housekeeping genes had similar properties. However, only eight housekeeping genes were identified in our data set. A further 277 genes (or 25\%) were included in 3,804 "wider definition" housekeeping genes, which are expressed at a more baseline or mid-level across all tissues and conditions (Eisenberg \& Levanon, 2013). Genes with frequent expression in several tissues could expand the range of life stages and situations where natural positive diversifying selection can modify allele frequencies in response to abiotic selection. Genes that are only expressed during embryonic development are aligned with thermal adaptation across latitudinal climate, elevation, and recurrent adaptation, pinpointing early development as a vulnerable stage for environmental positive diversifying selection (Sanger et al., 2018).

Whilst $18 \%$ of pEAGs were involved in the response to stress in experimental studies, we did not find evidence for an alignment between specific axes of environmental adaptation to specific types of stress response such as desert adaptation to heat stress response. This result supports the idea that functional genetic networks are partitioned into different types of functions covered by distinct gene sets, which then interlink with others for information exchange (Pancaldi et al., 2012), and supports the necessity of a generality of the organismal stress response to ensure viability in the face of multiple stressors (López-Maury et al., 2008). In general, while about 100-150 genes can be differentially expressed in response to any single stressor, the number of genes which responds to more than one stressor is much lower - genes responding to specific environmental stressors in comparison to total numbers of protein-coding genes are $0.5 \%-1.4 \%$ in Drosophila melanogaster and $0.4-0.7 \%$ in mammals, while genes responding across stressors are much lower with $0.5 \%$ in D. melanogaster and $0.14 \%-0.23 \%$ in mammals (de Nadal et al., 2011). These numbers also show that the $18 \%$ of stress- 
responsive genes found in our network must represent a large portion of the environmental stress response. As expected, HSPs were differentially expressed under all stressors (Feder \& Hofmann, 1999), and so were other genes functionally involved in the heat shock response. Besides HSPs, we here identified further little-studied pEAGs, that ubiquitously responded to all analyzed stressors (PKLR, AHSG, SQOR). Such genes may deserve further attention as possible biomarkers for environmental stress-related adaptation, as will be required under climate change conditions.

To conclude, we have shown that adaptations to abiotic environmental parameters are characterized by a high degree of genomic re-use and functional similarity, in line with the hypothesis of genomic constraint. Some interesting follow-on questions could include, whether selection in pEAGs in response to one type of environmental change happens via similar or different mutations, whether these mutation/selection events have the same effect size, and whether they are also conveying adaptation to different environmental conditions. As these genes typically have pleiotropic effects on the phenotype, it would also be interesting to study pEAGs in the context of linked traits. For example, an increase of cortisol can enhance the orange coloration in Zootoca vivipara, which is used in courtship (Andrade et al., 2019; Fitze et al., 2009). Although Lacertidae are thought to be excellent thermoregulators (Ortega et al., 2016; Sannolo et al., 2018), many lacertid lizard populations, especially those in humid montane localities, are currently undergoing population declines linked to climate change (Carneiro et al., 2017; Romero-Diaz et al., 2017). Studies are already underway that assess the future adaptive potential or "evolvability" of extant populations based on physiological parameters and distribution areas (Somero, 2010), and new genomic and transcriptomic datasets are rapidly accumulating (for Lacertidae, see Andrade et al., 2019; Kolora et al., 2019; Yurchenko et al., 2019). The genes identified in this study deserve concerted focus in future research aimed at evaluating current climate stress on populations at the molecular level, and whether it may lead to evolutionary adaptation in the future similar to, for example, Bay and colleagues' (Bay et al., 2017) approach to determine the likelihood of future heat adaptation in corals. We recommend that similar efforts in vertebrates focus on the genes and functions outlined in this contribution, and on the specific functional mutations of different alleles within these candidate genes. 


\section{Acknowledgements}

This work was partially supported by the Deutsche Forschungsgemeinschaft (DFG) to M.V. and J.M. (VE 247/11-1 / MU 1760/9-1). The University of Hull is thanked for funding the Molecular Stress in changing aquatic environments $\left(\mathrm{MolStress}_{2} \mathrm{O}\right)$ research cluster (supporting K.W.V., P.B.A., and L.F.); other cluster members are thanked for helpful discussions. KWV is supported by the Royal Society (RGS $\backslash$ R2 $\backslash 180033)$. Hervé Philippe (Station d'Ecologie Expérimentale CNRS, Moulis, France) is thanked for help with sequence analysis. Hernando Valero is thanked for assistance with OrthoDB. We acknowledge the Viper High Performance Computing facility of the University of Hull and its support team, specifically Seif Elnawasany (Microsoft) and Chris Collins (Hull). S.S. acknowledges an Allam PhD studentship and A.B. acknowledges a PhD scholarship within the Endothelial cells in chronic disease research cluster at $\mathrm{UoH}$.

\section{Author contributions}

KWV, MV, JM, KBS designed research, KWV, JGP, II, SK performed research, OJG, PP, APT contributed new reagents, or analytic tools, KWV, JGP, II, LF, AB, SFS, APT, PBA analyzed data, KWV and all co-authors wrote the paper 


\section{References}

Altenhoff, A. M., \& Dessimoz, C. (2009). Phylogenetic and functional assessment of orthologs inference projects and methods. PLoS Computational Biology, 5, e1000262.

Andrade, P., Pinho, C., Pérez I de Lanuza, G., Afonso, S., Brejcha, J., Rubin, C.-J., Wallerman, O., Pereira, P., Sabatino, S. J., Bellati, A., Pellitteri-Rosa, D., Bosakova, Z., Bunikis, I., Carretero, M. A., Feiner, N., Marsik, P., Paupério, F., Salvi, D., Soler, L., ... Carneiro, M. (2019).

Regulatory changes in pterin and carotenoid genes underlie balanced color polymorphisms in the wall lizard. Proceedings of the National Academy of Sciences of the United States of America, 116(12), 5633-5642.

Araújo, M. B., Ferri-Yáñez, F., Bozinovic, F., Marquet, P. A., Valladares, F., \& Chown, S. L. (2013). Heat freezes niche evolution. Ecology Letters, 16, 1206-1219.

Babonis, L. S., \& Martindale, M. Q. (2017). Phylogenetic evidence for the modular evolution of metazoan signalling pathways. Philosophical Transactions of the Royal Society of London. Series B, Biological Sciences, 372(1713). https://doi.org/10.1098/rstb.2015.0477

Bai, L., Liu, B., Ji, C., Zhao, S., Liu, S., Wang, R., Wang, W., Yao, P., Li, X., Fu, X., Yu, H., Liu, M., Han, F., Guan, N., Liu, H., Liu, D., Tao, Y., Wang, Z., Yan, S., ... Enqi Liu. (2018). Hypoxic and Cold Adaptation Insights from the Himalayan Marmot Genome. iScience. https://doi.org/10.1016/j.isci.2018.11.034

Bay, R. A., Rose, N. H., Logan, C. A., \& Palumbi, S. R. (2017). Genomic models predict successful coral adaptation if future ocean warming rates are reduced. Science Advances, 3(11), e1701413.

Bindea, G., Galon, J., \& Mlecnik, B. (2013). CluePedia Cytoscape plugin: pathway insights using integrated experimental and in silico data. Bioinformatics, 29(5), 661-663.

Bindea, G., Mlecnik, B., Hackl, H., Charoentong, P., Tosolini, M., Kirilovsky, A., Fridman, W.-H., Pagès, F., Trajanoski, Z., \& Galon, J. (2009). ClueGO: a Cytoscape plug-in to decipher functionally grouped gene ontology and pathway annotation networks. Bioinformatics , 25(8), 1091-1093.

Blount, Z. D., Lenski, R. E., \& Losos, J. B. (2018). Contingency and determinism in evolution: 
Replaying life's tape. In Science (Vol. 362, Issue 6415, p. eaam5979).

https://doi.org/10.1126/science.aam5979

Bond, M. R., \& Hanover, J. A. (2015). A little sugar goes a long way: the cell biology of O-GlcNAc. The Journal of Cell Biology, 208(7), 869-880.

Bourgeois, Y., Ruggiero, R. P., Manthey, J. D., \& Boissinot, S. (2019). Recent secondary contacts, linked selection and variable recombination rates shape genomic diversity in the model species Anolis carolinensis. Genome Biology and Evolution. https://doi.org/10.1093/gbe/evz110

Buttke, T. M., \& Sandstrom, P. A. (1994). Oxidative stress as a mediator of apoptosis. Immunology Today, 15(1), 7-10.

Campbell-Staton, S. C., Cheviron, Z. A., Rochette, N., Catchen, J., Losos, J. B., \& Edwards, S. V. (2017). Winter storms drive rapid phenotypic, regulatory, and genomic shifts in the green anole lizard. Science, 357(6350), 495-498.

Carneiro, D., Garcia-Munoz, E., Zagar, A., Pafilis, P., \& Carretero, M. A. (2017). Is ecophysiology congruent with the present-day relictual distribution of a lizard group? Evidence from preferred temperatures and water loss rates. The Herpetological Journal, 27(1), 47-56.

Chen, L., DeVries, A. L., \& Cheng, C. H. (1997). Convergent evolution of antifreeze glycoproteins in Antarctic notothenioid fish and Arctic cod. Proceedings of the National Academy of Sciences of the United States of America, 94(8), 3817-3822.

Dalle-Donne, I., Rossi, R., Milzani, A., Di Simplicio, P., \& Colombo, R. (2001). The actin cytoskeleton response to oxidants: from small heat shock protein phosphorylation to changes in the redox state of actin itself. Free Radical Biology \& Medicine, 31(12), 1624-1632.

de Nadal, E., Ammerer, G., \& Posas, F. (2011). Controlling gene expression in response to stress. Nature Reviews. Genetics, 12(12), 833-845.

Denver, R. J. (2009). Structural and functional evolution of vertebrate neuroendocrine stress systems. Annals of the New York Academy of Sciences, 1163, 1-16.

Diele-Viegas, L. M., \& Rocha, C. F. D. (2018). Unraveling the influences of Climate Change in Lepidosauria (Reptilia). Journal of Thermal Biology. https://doi.org/10.1016/j.jtherbio.2018.11.005 
Eisenberg, E., \& Levanon, E. Y. (2013). Human housekeeping genes, revisited. Trends in Genetics: TIG, 29(10), 569-574.

Feder, M. E., \& Hofmann, G. E. (1999). Heat-shock proteins, molecular chaperones, and the stress response: evolutionary and ecological physiology. Annual Review of Physiology, 61, 243-282.

Feiner, N., Rago, A., While, G. M., \& Uller, T. (2018). Signatures of selection in embryonic transcriptomes of lizards adapting in parallel to cool climate. Evolution; International Journal of Organic Evolution, 72(1), 67-81.

Fitze, P. S., Cote, J., San-Jose, L. M., Meylan, S., Isaksson, C., Andersson, S., Rossi, J.-M., \& Clobert, J. (2009). Carotenoid-based colours reflect the stress response in the common lizard. PloS One, 4(4), e5111.

Futuyma, D. J. (2010). Evolutionary constraint and ecological consequences. Evolution; International Journal of Organic Evolution, 64(7), 1865-1884.

Garcia-Porta, J., Irisarri, I., Kirchner, M., Rodríguez, A., Kirchhof, S., Brown, J. L., MacLeod, A., Turner, A. P., Ahmadzadeh, F., Albaladejo, G., Crnobrnja-Isailovic, J., De la Riva, I., Fawzi, A., Galán, P., Göçmen, B., Harris, D. J., Jiménez-Robles, O., Joger, U., Jovanović Glavaš, O., ... Wollenberg Valero, K. C. (2019). Environmental temperatures shape thermal physiology as well as diversification and genome-wide substitution rates in lizards. Nature Communications, 10(1), 4077.

Hancock, A. M., Witonsky, D. B., Gordon, A. S., Eshel, G., Pritchard, J. K., Coop, G., \& Di Rienzo, A. (2008). Adaptations to climate in candidate genes for common metabolic disorders. PLoS Genetics, 4(2), e32.

Han, J.-D. J., Bertin, N., Hao, T., Goldberg, D. S., Berriz, G. F., Zhang, L. V., Dupuy, D., Walhout, A. J. M., Cusick, M. E., Roth, F. P., \& Vidal, M. (2004). Evidence for dynamically organized modularity in the yeast protein-protein interaction network. Nature, 430(6995), 88-93.

Hayes, J. D., Dinkova-Kostova, A. T., \& Tew, K. D. (2020). Oxidative Stress in Cancer. Cancer Cell, $38(2), 167-197$.

Hsiao, L. L., Dangond, F., Yoshida, T., Hong, R., Jensen, R. V., Misra, J., Dillon, W., Lee, K. F., Clark, K. E., Haverty, P., Weng, Z., Mutter, G. L., Frosch, M. P., MacDonald, M. E., Milford, E. 
L., Crum, C. P., Bueno, R., Pratt, R. E., Mahadevappa, M., ... Gullans, S. R. (2001). A compendium of gene expression in normal human tissues. Physiological Genomics, 7(2), $97-$ 104.

Jensen, L. J., Gupta, R., Blom, N., Devos, D., Tamames, J., Kesmir, C., Nielsen, H., Staerfeldt, H. H., Rapacki, K., Workman, C., Andersen, C. A. F., Knudsen, S., Krogh, A., Valencia, A., \& Brunak, S. (2002). Prediction of human protein function from post-translational modifications and localization features. Journal of Molecular Biology, 319(5), 1257-1265.

Klose, M. K., \& Robertson, R. M. (2004). Stress-induced thermoprotection of neuromuscular transmission. Integrative and Comparative Biology, 44(1), 14-20.

Kolora, S. R. R., Weigert, A., Saffari, A., Kehr, S., Walter Costa, M. B., Spröer, C., Indrischek, H., Chintalapati, M., Lohse, K., Doose, G., Overmann, J., Bunk, B., Bleidorn, C., Grimm-Seyfarth, A., Henle, K., Nowick, K., Faria, R., Stadler, P. F., \& Schlegel, M. (2019). Divergent evolution in the genomes of closely related lacertids, Lacerta viridis and L. bilineata, and implications for speciation. GigaScience, 8(2). https://doi.org/10.1093/gigascience/giy160

Kosakovsky Pond, S. L., Frost, S. D. W., \& Muse, S. V. (2005). HyPhy: hypothesis testing using phylogenies. Bioinformatics , 21(5), 676-679.

Kowalczyk, A., Meyer, W. K., Partha, R., Mao, W., Clark, N. L., \& Chikina, M. (2019).

RERconverge: an R package for associating evolutionary rates with convergent traits. Bioinformatics (Oxford, England), 35(22), 4815-4817.

Kowalczyk, A., Partha, R., Clark, N. L., \& Chikina, M. (2020). Pan-mammalian analysis of molecular constraints underlying extended lifespan. eLife, 9. https://doi.org/10.7554/eLife.51089

Kriventseva, E. V., Kuznetsov, D., Tegenfeldt, F., Manni, M., Dias, R., Simão, F. A., \& Zdobnov, E. M. (2019). OrthoDB v10: sampling the diversity of animal, plant, fungal, protist, bacterial and viral genomes for evolutionary and functional annotations of orthologs. Nucleic Acids Research, 47(D1), D807-D811.

Lahti, D. C., Johnson, N. A., Ajie, B. C., Otto, S. P., Hendry, A. P., Blumstein, D. T., Coss, R. G., Donohue, K., \& Foster, S. A. (2009). Relaxed selection in the wild. Trends in Ecology \& Evolution, 24(9), 487-496. 
Lê, S., Josse, J., Husson, F., \& Others. (2008). FactoMineR: an R package for multivariate analysis. Journal of Statistical Software, 25(1), 1-18.

Logan, C. A., \& Buckley, B. A. (2015). Transcriptomic responses to environmental temperature in eurythermal and stenothermal fishes. The Journal of Experimental Biology, 218(Pt 12), 19151924.

López-Maury, L., Marguerat, S., \& Bähler, J. (2008). Tuning gene expression to changing environments: from rapid responses to evolutionary adaptation. Nature Reviews. Genetics, 9(8), $583-593$.

Losos, J. B. (2011). Convergence, adaptation, and constraint. Evolution; International Journal of Organic Evolution, 65(7), 1827-1840.

Lukk, M., Kapushesky, M., Nikkilä, J., Parkinson, H., Goncalves, A., Huber, W., Ukkonen, E., \& Brazma, A. (2010). A global map of human gene expression. Nature Biotechnology, 28(4), 322324.

Martin, T. L., \& Huey, R. B. (2008). Why “suboptimal” is optimal: Jensen's inequality and ectotherm thermal preferences. The American Naturalist, 171(3), E102-E118.

Morin, P., Jr, \& Storey, K. B. (2009). Mammalian hibernation: differential gene expression and novel application of epigenetic controls. The International Journal of Developmental Biology, 53(2-3), $433-442$.

Munoz, M. M., Stimola, M. A., Algar, A. C., Conover, A., Rodriguez, A. J., Landestoy, M. A., Bakken, G. S., \& Losos, J. B. (2014). Evolutionary stasis and lability in thermal physiology in a group of tropical lizards. Proceedings of the Royal Society of London B: Biological Sciences, 281(1778), 20132433.

Niu, Y., DesMarais, T. L., Tong, Z., Yao, Y., \& Costa, M. (2015). Oxidative stress alters global histone modification and DNA methylation. Free Radical Biology \& Medicine, 82, 22-28.

Ortega, Z., Mencía, A., \& Pérez-Mellado, V. (2016). The peak of thermoregulation effectiveness: Thermal biology of the Pyrenean rock lizard, Iberolacerta bonnali (Squamata, Lacertidae). Journal of Thermal Biology, 56, 77-83.

Pancaldi, V., Saraç, O. S., Rallis, C., McLean, J. R., Převorovský, M., Gould, K., Beyer, A., \& 
Bähler, J. (2012). Predicting the fission yeast protein interaction network. G3 , 2(4), 453-467.

Pavlicev, M., \& Wagner, G. P. (2012). A model of developmental evolution: selection, pleiotropy and compensation. Trends in Ecology \& Evolution, 27(6), 316-322.

Pedersen, S. F., Hoffmann, E. K., \& Mills, J. W. (2001). The cytoskeleton and cell volume regulation. Comparative Biochemistry and Physiology. Part A, Molecular \& Integrative Physiology, 130(3), $385-399$.

Plotnikov, A., Zehorai, E., Procaccia, S., \& Seger, R. (2011). The MAPK cascades: signaling components, nuclear roles and mechanisms of nuclear translocation. Biochimica et Biophysica Acta, 1813(9), 1619-1633.

Porcelli, D., Butlin, R. K., Gaston, K. J., Joly, D., \& Snook, R. R. (2015). The environmental genomics of metazoan thermal adaptation. Heredity, 114(5), 502-514.

Pozhitkov, A. E., Neme, R., Domazet-Lošo, T., Leroux, B. G., Soni, S., Tautz, D., \& Noble, P. A. (2017). Tracing the dynamics of gene transcripts after organismal death. Open Biology, 7(1). https://doi.org/10.1098/rsob.160267

Qiu, Q., Zhang, G., Ma, T., Qian, W., Wang, J., Ye, Z., Cao, C., Hu, Q., Kim, J., Larkin, D. M., Auvil, L., Capitanu, B., Ma, J., Lewin, H. A., Qian, X., Lang, Y., Zhou, R., Wang, L., Wang, K., ... Liu, J. (2012). The yak genome and adaptation to life at high altitude. Nature Genetics, 44(8), 946-949.

Rodríguez, A., Rusciano, T., Hamilton, R., Holmes, L., Jordan, D., \& Wollenberg Valero, K. C. (2017). Genomic and phenotypic signatures of climate adaptation in an Anolis lizard. Ecology and Evolution, 7, 6390-6403.

Romero-Diaz, C., Breedveld, M. C., \& Fitze, P. S. (2017). Climate effects on growth, body condition, and survival depend on the genetic characteristics of the population. The American Naturalist, $190(5), 649-662$.

Salverda, M. L. M., Dellus, E., Gorter, F. A., Debets, A. J. M., van der Oost, J., Hoekstra, R. F., Tawfik, D. S., \& de Visser, J. A. G. M. (2011). Initial mutations direct alternative pathways of protein evolution. PLoS Genetics, 7(3), e1001321.

Sanger, T. J., Kyrkos, J., Lachance, D. J., Czesny, B., \& Stroud, J. T. (2018). The effects of thermal 
stress on the early development of the lizard Anolis sagrei. Journal of Experimental Zoology. Part A, Ecological and Integrative Physiology. https://doi.org/10.1002/jez.2185

Sannolo, M., Barroso, F. M., \& Carretero, M. A. (2018). Physiological differences in preferred temperatures and evaporative water loss rates in two sympatric lacertid species. Zoology, 126, $58-64$.

Shannon, P., Markiel, A., Ozier, O., Baliga, N. S., Wang, J. T., Ramage, D., Amin, N., Schwikowski, B., \& Ideker, T. (2003). Cytoscape: a software environment for integrated models of biomolecular interaction networks. Genome Research, 13(11), 2498-2504.

Simonson, T. S. (2015). Altitude Adaptation: A Glimpse Through Various Lenses. High Altitude Medicine \& Biology, 16(2), 125-137.

Smith, M. D., Wertheim, J. O., Weaver, S., Murrell, B., Scheffler, K., \& Kosakovsky Pond, S. L. (2015). Less is more: an adaptive branch-site random effects model for efficient detection of episodic diversifying selection. Molecular Biology and Evolution, 32(5), 1342-1353.

Sokolova, I. (2018). Mitochondrial adaptations to variable environments and their role in animals' stress tolerance. Integrative and Comparative Biology. https://oi.org/10.1093/icb/icy017.

Somero, G. N. (2010). The physiology of climate change: how potentials for acclimatization and genetic adaptation will determine "winners" and "losers." The Journal of Experimental Biology, 213(6), 912-920.

Sun, Y.-B., Fu, T.-T., Jin, J.-Q., Murphy, R. W., Hillis, D. M., Zhang, Y.-P., \& Che, J. (2018). Species groups distributed across elevational gradients reveal convergent and continuous genetic adaptation to high elevations. Proceedings of the National Academy of Sciences of the United States of America, 115(45), E10634-E10641.

Suzuki, R., \& Shimodaira, H. (2006). Pvclust: an R package for assessing the uncertainty in hierarchical clustering. Bioinformatics , 22(12), 1540-1542.

Suzuki, R., \& Shimodaira, H. (2013). pvclust: An R package for hierarchical clustering with p-values. Bioinformatics , 22, 1-7.

Tan, Z., Chan, Y. J. A., Chua, Y. J. K., Rutledge, S. D., Pavelka, N., Cimini, D., \& Rancati, G. (2019). Environmental stresses induce karyotypic instability in colorectal cancer cells. 
Molecular Biology of the Cell, 30(1), 42-55.

Therkildsen, N. O., Wilder, A. P., Conover, D. O., Munch, S. B., Baumann, H., \& Palumbi, S. R. (2019). Contrasting genomic shifts underlie parallel phenotypic evolution in response to fishing. In Science (Vol. 365, Issue 6452, pp. 487-490). https://doi.org/10.1126/science.aaw7271 Turelli, M. (1985). Effects of pleiotropy on predictions concerning mutation-selection balance for polygenic traits. Genetics, 111(1), 165-195.

Wagner, J. T., Singh, P. P., Romney, A. L., Riggs, C. L., Minx, P., Woll, S. C., Roush, J., Warren, W. C., Brunet, A., \& Podrabsky, J. E. (2018). The genome of Austrofundulus limnaeus offers insights into extreme vertebrate stress tolerance and embryonic development. BMC Genomics, 19(1), 155 .

Wang, J. Z., Du, Z., Payattakool, R., Yu, P. S., \& Chen, C.-F. (2007). A new method to measure the semantic similarity of GO terms. Bioinformatics , 23(10), 1274-1281.

Wertheim, J. O., Murrell, B., Smith, M. D., Kosakovsky Pond, S. L., \& Scheffler, K. (2015). RELAX: detecting relaxed selection in a phylogenetic framework. Molecular Biology and Evolution, 32(3), 820-832.

White, C. R., Alton, L. A., \& Frappell, P. B. (2012). Metabolic cold adaptation in fishes occurs at the level of whole animal, mitochondria and enzyme. Proceedings of the Royal Society B: Biological Sciences, 279(1734), 1740-1747.

White, R. J., Collins, J. E., Sealy, I. M., Wali, N., Dooley, C. M., Digby, Z., Stemple, D. L., Murphy, D. N., Billis, K., Hourlier, T., Füllgrabe, A., Davis, M. P., Enright, A. J., \& Busch-Nentwich, E. M. (2017). A high-resolution mRNA expression time course of embryonic development in zebrafish. eLife, 6. https://doi.org/10.7554/eLife.30860

Wollenberg Valero, K. C. (2020). Aligning functional network constraint to evolutionary outcomes. BMC Evolutionary Biology, 20(1), 58.

Wollenberg Valero, K. C., Pathak, R., Prajapati, I., Bankston, S., Thompson, A., Usher, J., \& Isokpehi, R. D. (2014). A candidate multimodal functional genetic network for thermal adaptation. PeerJ, 2, e578.

Wu, H., Guang, X., Al-Fageeh, M. B., Cao, J., Pan, S., Zhou, H., Zhang, L., Abutarboush, M. H., 
Xing, Y., Xie, Z., Alshanqeeti, A. S., Zhang, Y., Yao, Q., Al-Shomrani, B. M., Zhang, D., Li, J., Manee, M. M., Yang, Z., Yang, L., ... Wang, J. (2014). Camelid genomes reveal evolution and adaptation to desert environments. Nature Communications, 5, 5188.

Xu, H., Chen, X., Xu, X., Shi, R., Suo, S., Cheng, K., Zheng, Z., Wang, M., Wang, L., Zhao, Y., Tian, B., \& Hua, Y. (2016). Lysine Acetylation and Succinylation in HeLa Cells and their Essential Roles in Response to UV-induced Stress. Scientific Reports, 6, 30212.

Yang, W., Qi, Y., \& Fu, J. (2014). Exploring the genetic basis of adaptation to high elevations in reptiles: a comparative transcriptome analysis of two toad-headed agamas (genus Phrynocephalus). PloS ONE, 9(11), e112218.

Yi, H., Xue, L., Guo, M.-X., Ma, J., Zeng, Y., Wang, W., Cai, J.-Y., Hu, H.-M., Shu, H.-B., Shi, Y.B., \& Li, W.-X. (2010). Gene expression atlas for human embryogenesis. FASEB Journal: Official Publication of the Federation of American Societies for Experimental Biology, 24(9), $3341-3350$.

Yudin, N. S., Larkin, D. M., \& Ignatieva, E. V. (2017). A compendium and functional characterization of mammalian genes involved in adaptation to Arctic or Antarctic environments. BMC Genetics, 18(Suppl 1), 111.

Yu, G., Li, F., Qin, Y., Bo, X., Wu, Y., \& Wang, S. (2010). GOSemSim: an R package for measuring semantic similarity among GO terms and gene products. Bioinformatics , 26(7), 976-978.

Yu, J., \& Auwerx, J. (2010). Protein deacetylation by SIRT1: an emerging key post-translational modification in metabolic regulation. Pharmacological Research: The Official Journal of the Italian Pharmacological Society, 62(1), 35-41.

Yurchenko, A. A., Recknagel, H., \& Elmer, K. R. (2019). Chromosome-level assembly of the common lizard (Zootoca vivipara) genome. In Cold Spring Harbor Laboratory (p. 520528). https://doi.org/10.1101/520528

Zhuang, X., Yang, C., Murphy, K. R., \& Cheng, C.-H. C. (2019). Molecular mechanism and history of non-sense to sense evolution of antifreeze glycoprotein gene in northern gadids. Proceedings of the National Academy of Sciences of the United States of America. https://doi.org/10.1073/pnas.1817138116 